\title{
PDEF downregulates stathmin expression in prostate cancer
}

\author{
YAMINI SABHERWAL ${ }^{1}$, NITIN MAHAJAN ${ }^{1}$, DONALD L. HELSETH ${ }^{2}$, \\ MAYUMI GASSMANN ${ }^{3}$, HEIDI SHI ${ }^{1}$ and MING ZHANG ${ }^{1}$ \\ ${ }^{1}$ Department of Molecular Pharmacology and Biological Chemistry, Robert H. Lurie Cancer Center, \\ Northwestern University, Feinberg School of Medicine, Chicago, IL 60611; ${ }^{2} \mathrm{CBC} /$ RRC Proteomics \\ and Informatics Services Facility, University of Illinois at Chicago, Chicago, IL 60612; \\ ${ }^{3}$ University of Illinois, Urbana-Champaign, Urbana, IL 60180, USA
}

Received December 14, 2011; Accepted January 30, 2012

DOI: 10.3892/ijo.2012.1392

\begin{abstract}
The Ets proteins are a family of transcription factors characterized by an evolutionarily conserved DNA binding domain that controls key cellular processes. Prostate-derived Ets transcription factor (PDEF), a member of the Ets family, is reported to be present in tissues with high epithelial content, notably breast and prostate. However, the role of PDEF in cancer development is not fully understood. To gain insight into the molecular mechanisms associated with prostate cancer progression, we employed iTRAQ labeling followed by mass spectrometric (MS) analysis to identify candidate proteins that are differentially expressed in prostate cancer cells with or without PDEF. To this end, we overexpressed PDEF in PC3 human prostate cells using a tetracycline inducible system (Tet-On). Many differentially expressed proteins which play important roles in various cellular and biological processes were identified. Among them, stathmin (STMN), which is a microtubule (MT)-destabilizing protein, was found to be downregulated in multiple analyses. We demonstrated that re-expression of STMN reversed the antitumor properties of PDEF in PDEF-overexpressing PC3 cells. Using in vitro functional assays, we showed that STMN overexpression counteracted PDEF's effects against cell proliferation, colony formation and tumor migration. Similar results were further confirmed with the prostate cancer cell line CWR22rv1. In conclusion, many differentially expressed proteins were identified and STMN was found to be downregulated by PDEF.
\end{abstract}

Correspondence to: Dr Ming Zhang, Department of Molecular Pharmacology and Biological Chemistry, Northwestern University, Feinberg School of Medicine, Chicago, IL 60611, USA

E-mail: m-zhang@northwestern.edu

Abbreviations: iTRAQ, isobaric tags of relative and absolute quantitation; STMN, stathmin; MT, microtubule; PDEF, prostatederived Ets transcription factor; MS, mass spectrometry; Tet-On, tetracycline inducible system

Key words: prostate cancer, prostate-derived Ets transcription factor, iTRAQ labeling, mass spectrometry, stathmin
These results suggest that PDEF may inhibit prostate cancer progression by transcriptional downregulation of oncogenic STMN expression. Analyzing the association among differentially expressed proteins may provide a basis to better understand the molecular mechanisms underlying the process of cancer progression and development and further aid in designing therapeutics in the future.

\section{Introduction}

Prostate cancer is the most common cancer diagnosed among men and the second leading cause of death in American men, behind only lung cancer. The American Cancer Society (ACS) estimates about 1 man in 36 will die of prostate cancer (1). Although screening for prostate cancer, based on prostate specific antigen (PSA) has revolutionized early detection and diagnosis of the disease, the challenge that clinicians face are to determine which patients progress to aggressive disease $(2,3)$. In order to determine the possibility of disease progression and ability to manage patient outcomes, it is necessary to better understand the molecular processes underlying the disease development and progression. Gene regulation is an important process in maintaining the integrity of cells for their proper growth and survival. Improper regulation of genes can lead to various diseases like cancer. The Ets family of transcription factors has been long investigated for their role in genetic loss of cellular homeostasis which results in development of various cancers. So far, 25 human and 26 murine Ets family members have been reported (4). Ets family of proteins share a conserved winged helix-turn-helix DNA binding domain called the Ets domain which recognizes unique DNA sequences containing GGAA/T. Eleven Ets proteins have a pointed (PNT) domain, important in protein-protein association. Ets proteins control many important cellular processes, including proliferation, differentiation, angiogenesis and apoptosis (4-11). Prostate-derived Ets transcription factor (PDEF), a member of the Ets family has been widely investigated for its role in cancer development and progression. Originally identified from the prostate epithelium, PDEF controls the expression of PSA, a specific marker for prostate cancer (12). Immunohistochemical analysis has shown the presence of PDEF in all high-epithelial content tissues such as breast and 
prostate $(13,14)$. Early reports showed that PDEF was a tumor promoter $(13,14)$. However, recent studies support its role as a tumor suppressor based on differing model systems and varying experimental conditions $(14,15-18)$.

In order to gain insight into the function of PDEF, in the present study we used isobaric tags for relative and absolute quantitation (iTRAQ)-labeling coupled with mass spectrometry (MS) to identify differentially expressed proteins upon PDEF induction using a Tet-On system in prostate cancer cells (19). This study is the first to report the use of iTRAQ labeling MS to identify differentially expressed proteins upon PDEF induction and validate the results with in vitro functional assays $(20,21)$. iTRAQ coupled with MS has significant advantages over other methods due to its capability of multiplexing samples in one experimental setup $(20,22)$. Another positive aspect includes unbiased peptide labeling, since iTRAQ tags label lysine side groups and all free amino-terminal groups of the peptides present in the sample (23). Keeping this background in mind, we thought it would be worth exploring the PDEF proteome using iTRAQ labeling with MS and validate the results with in vitro assays to study the role of PDEF in prostate cancer progression. To date only one study has been done in MDA-MB-231 human breast cancer cells analyzing interacting protein complexes to overexpressed PDEF (24). Cho et al used adenoviral-mediated gene delivery system to overexpress PDEF and LC-MS/MS analysis to identify protein complexes. They identified 121 proteins and their findings suggested PDEF may be regulated by ERBB2 or EGFR-activated signaling pathways in breast cancer (24). No proteomics research work has been done coupled with in vitro functional assays with PDEF in prostate cells. Our research is the first to utilize iTRAQ labeling to identify differentially expressed proteins upon PDEF induction in prostate cancer cells and verify the results with in vitro assays to study the role of PDEF. We identified 115 proteins, of which 35 were upregulated and 80 were downregulated in the two sets of prostate cancer cells. Stathmin (STMN), a microtubule (MT)-destabilizing protein, was found downregulated mulitple times in the presence of PDEF in the analyzed sample sets and therefore took our attention. Further, the in vitro functional assays such as proliferation, migration and colony formation used to verify the results confirmed correlation of PDEF with the downregulation of STMN in PDEF overexpressing prostate cancer cells (PC3 and CWR22rv1).

\section{Materials and methods}

Chemicals and reagents. The following primary antibodies were used: hPDEF rabbit polyclonal antibody (PDEF N-terminal construct obtained from the Laboratory of Dennis K. Watson from the Medical University of South Carolina antibody made by Proteintech, IL), STMN antibody (EMD Chemicals, Inc.) and anti-actin antibody (Santa Cruz Biotechnology, Inc.); secondary antibodies used for western blots were: horseradish peroxidaseconjugated anti-goat (Roche Applied Science), anti-mouse (Bio-Rad) and anti-rabbit Santa Cruz Biotechnology, Inc.); and G418 Sulfate/Neomycin (Invitrogen, Inc.) and Puromycin (Sigma, Inc.) were used for selection of stable clones.

Cell culture. The human prostate cancer cell lines PC3 and CWR22rv1 and stable transfectants (ATCC) were maintained in RPMI-1640 (Invitrogen, Inc.) supplemented with 5\% fetal bovine serum (FBS; Invitrogen, Inc.) and antibiotic liquid (penicillin and streptomycin; Invitrogen, Inc.) at $37^{\circ} \mathrm{C}$ under $5 \% \mathrm{CO}_{2}$.

A Tet-On prostate cancer cell line was used (19) and PDEF expression was routinely induced by using doxycycline (DOX) (RPI, Inc.) at a concentration of $1 \mu \mathrm{g}$ for $24 \mathrm{~h}$ in PC3 cells. This system can induce activation of target gene (i.e., PDEF in our study) in a stringent, reversible, specific manner to study underlying biological and pathological processes (25).

RT-PCR analysis. Total cellular RNA was isolated from cell lines using the TRIzol Reagent (Invitrogen, Inc.) according to the manufacturer's instructions. A two-step RT-PCR was used to analyze mRNA expression of STMN and GAPDH genes. A cDNA was created using oligo(dT) primer and the Moloney murine leukemia virus reverse transcriptase enzyme according to manufacturer's instructions (Invitrogen, Inc.). Standard PCR techniques were then conducted with gene-specific primers. The primer set used for PDEF amplification was as follows: forward primer 5'-GGT GAA AGA ACT GGA GAA GCG-3' and reverse primer 5'-GTG CTT ATC CTT CTC TCG C-3'.

Transfection. PDEF cDNA coding sequence was amplified by RT-PCR and cloned into a eukaryotic expression vector pcDNA3.1. PC3 and CWR22rv1 cells were transfected with this recombinant plasmid pcDNA3.1 hPDEF or control vector alone by Lipofectamine 2000 (Invitrogen, Inc.). The stable transfectants were selected with G418 medium. PDEF protein expression was detected with western blot analysis in the transfected cell lines. STMN cDNA bacterial stock was obtained from Thermo Scientific, Inc. and the STMN cDNA was cloned into $\mathrm{pEF}$ puromycin expression vector. $\mathrm{PC} 3 \mathrm{pcDNA} 3.1 \mathrm{hPDEF}$ and CWR22rv1 pcDNA3.1 hPDEF cells were transfected with pEF-STMN vector alone by Lipofectamine 2000. The stable transfectants were selected with puromycin medium. From now on the respective stable transfectants will be referred to as follows: PC3/CWR22rv1 pcDNA3.1 control, PC3/CWR22rv1 control; PC3/CWR22rv1 pcDNA3.1 hPDEF, PC3/CWR22rv1 hPDEF; PC3/CWR22rv1 pcDNA3.1 hPDEF+pEF STMN, PC3/CWR22rv1 hPDEF+STMN.

iTRAQ protein sample preparation. Protein extraction was performed according to Applied Biosystems (now AB-SCIEX) protocol available from their website (http://www.absciex. com/). Briefly, adherent cells were detached using trypsin then washed twice with phosphate-buffered saline (PBS). Pelleted cells were homogenized in radioimmunoprecipitation assay buffer (RIPA) buffer containing a cocktail of HALT protease inhibitors (Thermo Scientific, Inc.). The cells were vortexed and kept on ice for $30 \mathrm{~min}$ and then centrifuged at $10000 \mathrm{x} \mathrm{g}$ for $20 \mathrm{~min}$ at $40^{\circ} \mathrm{C}$. The protein concentration of the supernatants was determined by the bicinchoninic acid (BCA) protein assay kit (Thermo Scientific, Inc.) and stored in aliquots at $-20^{\circ} \mathrm{C}$ until use.

Isobaric labeling. One hundred micrograms of protein from the [PC3 tetracycline (Tet-On) control-uninduced and PC3 Tet-On PDEF-induced] cells were subjected to trichloroacetic acid (TCA) precipitation followed by several washes in acetone 
to remove Tris and detergents. The pellet was reconstituted by resuspending into $20 \mu \mathrm{l}$ of $8 \mathrm{M}$ urea/500 mM triethylammonium bicarbonate (TEAB). Subsequently, the resuspended proteins were reduced, alkylated with iodoacetamide and digested with trypsin (Promega, Madison, WI) according to manufacturer's protocol (AB SCIEX, Framingham, MA, USA). Samples were iTRAQ labeled as follows: PC3 Tet-On controluninduced was labeled with the 114 reagent and PC3 Tet-On PDEF-induced was labeled with the 117 reagent, samples were pooled and a preparative desalting was done using an mRP C18 reverse phase column (Agilent Technologies). iTRAQ labeling experiment was repeated for reproducibility. Peptide fractions were collected $(\sim 15 \mathrm{ml}$ each) and concentrated in a vacuum concentrator to $\sim 0.3 \mathrm{ml}$ prior to fractionation.

OFFGEL fractionation. Peptides were separated using an Agilent 3100 OFFGEL Fractionator (Agilent, G3100AA). The OFFGEL isoelectric fractionation was run with commercially available IPG Dry strips pI 3-10 (GE Healthcare) in dilute glycerol only (no amphophiles added) with the standard 12-well peptide fractionation program until $20 \mathrm{kVh}$ was accumulated ( $\sim 24 \mathrm{~h}$ ), then each of the 12 fractions was either injected directly onto the LTQ (Thermo Fisher) linear ion trap mass spectrometer or fractions were desalted using C18 Porous R2 (Applied Biosystems) prior to loading onto the LTQ (26).

Mass spectrometric analysis and data processing. Individual OFFGEL fractions were infused separately onto a Thermo Electron Finnigan linear ion trap mass spectrometer (LTQ) operated in positive ion mode via a Dionex U-3000 Ultimate nano LC system running. Peptides were separated on a $\mathrm{C} 18$ column (Agilent Zorbax 300SB-C18, $3.5 \mu \mathrm{m}, 75 \mu \mathrm{m} \times 150 \mathrm{~mm}$ ) at a flow rate of $250 \mathrm{nl} / \mathrm{min}$ eluted with a linear gradient of $10-60 \%$ solution B (95\% ACN, $0.1 \%$ formic acid) over $80 \mathrm{~min}$. A survey full scan $(\mathrm{m} / \mathrm{z}=400-2000)$ and the five most intense ions were selected for a zoom scan to determine the charge state, after which MS/MS was triggered in Pulsed-Q Dissociation mode (PQD) with minimum signal required (1000), isolation width 2.0, normalized collision energy 31.0, activation Q 0.600 and activation time of 0.400 . PQD mode is critical in order to visualize the low $\mathrm{m} / \mathrm{z}$ reporter ions from the iTRAQ reagents used for relative quantitation. Mass spectrometry data were acquired with Xcalibur software version 2.0 SR2. Each resulting RAW file was extracted using Readw.exe (version 4.0.2, Institute for Systems Biology) to produce mzXML files and with DTASuperCharge (University of Southern Denmark) to give MGF files. Individual MGF files were merged using a text editor for searching. Protein identification was performed using a Sorcerer search appliance (SageN) running the SEQUEST algorithm using the IPI Human database v3.50 using iTRAQ ( $\mathrm{K}$ and $\mathrm{N}$-terminal) as fixed modifications and oxidation of methionine and carbamidomethylation of cysteine as variable modifications using a peptide tolerance of 2.0 Da and fragment tolerance of 0.6 Da. These results were visualized using Scaffold 2.1 (Proteome Software). Proteins were also identified by searching the merged MGF file against Mascot 2.2 search engine (MatrixScience) using the IPI Human database v3.50, using iTRAQ 4-plex quantitation (quantitation methods were modified for carbamidomethylation instead of methylthio). The resulting .DAT file was extracted to generate the .CSV result file for further analysis.
The Mascot search engine returns the relative ratio to 114 , so $117 / 114<1$ corresponds to downregulation and 117/114>1 corresponds to upregulation. The searches were also run using a decoy database to give us an estimate of false positive rate against the IPI human database. The 114 and 117 ratios were compensated during the analysis.

MTT in vitro cell proliferation assay. Cells were seeded at 1000 cells/well in 96-well dishes and allowed to grow at $37^{\circ} \mathrm{C}$ with $5 \% \mathrm{CO}_{2}$. At each time point MTT [3-(4,5-dimethylthiazolyl-2)-2,5-diphenyltetrazolium bromide] reagent $(5 \mathrm{mg} / \mathrm{ml})$ was added in a volume of $10 \mu \mathrm{l}$ per well and incubated at $37^{\circ} \mathrm{C}$ with $5 \% \mathrm{CO}_{2}$ for $3 \mathrm{~h}$. The media were aspirated and $100 \mu \mathrm{l}$ of DMSO was added and mixed until a uniform purple color formed. The cell samples were measured using a plate reader at $570 \mathrm{~nm}$. Assays were performed in triplicates.

Soft agar colony formation assay. As described previously (16), 6-well dishes were plated with bottom agar (1.3 $\mathrm{ml} \mathrm{1.8 \%}$ agarose and $0.3 \mathrm{ml}$ of $2 \mathrm{X}$ DMEM) for 20-30 min. Cells were mixed with the top agar $(0.3 \mathrm{ml} 1.8 \%$ agarose and $0.7 \mathrm{ml}$ DMEM) at a concentration of $5 \times 10^{4}$ cells per $\mathrm{ml}$ and plated in 6-well dishes and allowed to solidify. The cells were grown at $37^{\circ} \mathrm{C}$ for 2 weeks, cells stained for $1 \mathrm{~h}$ with $0.05 \%$ crystal violet and the number of purple colored colonies were tabulated. Three independent experiments (in duplicates) were averaged for graphical representation.

Wound healing migration assay. Cells were seeded in 6-well plates in duplicates so that the cells were $90 \%$ confluent the next day. Cells were seeded in RPMI-1640 medium containing $5 \%$ FBS overnight. The next day, monolayer of cells was washed with PBS and a wound was created with a pipette tip. Cells were washed again with PBS to remove any leftover cells after the wound was created. The wound was allowed to close in serum-free medium for $24 \mathrm{~h}$. Images were taken at a magnification of x100 and at time points of 0 and $24 \mathrm{~h}$ at the same position the wound was created. Cells which migrated towards the wound for closure were counted in duplicate wells and averaged: results represented as percent migrated cells.

\section{Results}

Selection criteria of peptides for quantitation of iTRAQ analysis. In order to obtain reliable analytical measurements, the experiments were repeated more than once. Based on the selection criteria pre-set, all peptides were used for quantitation with: i) a significance threshold $\mathrm{p}<0.05$, ii) require bold red, as per Mascot's search criteria (http://www.matrixscience. com) and iii) minimum 2 peptides/protein. A total of 115 proteins were identified and quantified ( $\geq 95 \%$ confidence; $\geq 2$ peptides/protein), 35 proteins were upregulated $(>1.0)$ and 80 downregulated $(<1.0)$ in uninduced cells relative to induced PC3 Tet-On cells.

Identification of differentially expressed proteins upon PDEF induction. To identify proteins differentially expressed upon PDEF induction, PC3 Tet-On system was used as described previously (25). The western blot analysis of the induced sample (Fig. 1A) showed a 3-fold higher expression of PDEF 
Table I. Differentially expressed proteins upon prostate-derived Ets transcription factor induction.

$\begin{array}{ll}\text { iTRAQ ratio } & \text { Protein name }\end{array}$

\section{Upregulated proteins}

1.049

1.049

1.049

1.626

1.626

1.307

1.307

1.050

1.395

1.395

1.395

1.141

1.141

1.088

1.344

1.344

1.344

1.071

1.071

1.599

1.309

1.750

1.150

1.150

1.045

1.045

1.336

1.198

1.148

1.046

1.082

1.082

1.082

1.078

Downregulated proteins

0.667

0.812

0.764

0.829

0.829

0.916

0.970

0.574

0.569

0.835

0.666

0.666

0.666
HNRNPU heterogeneous nuclear ribonucleoprotein $U$ isoform a

TUBA1A tubulin $\alpha-1$ A chain

TUBA1C $\alpha-1 \mathrm{C}$ chain

TUBA1C $29 \mathrm{kDa}$ protein

TUBB3 tubulin, $\beta, 4$

cDNA FLJ56903, highly similar to tubulin $\beta-7$ chain

TUBB tubulin $\beta$ chain

TUBB2A tubulin $\beta$-2A chain

PHB prohibitin

CTTN protein

CTTN Src substrate cortactin

CTTN putative uncharacterized protein CTTN

CAST calpastatin isoform e

CAST calpastatin isoform a

TAGLN2 $24 \mathrm{kDa}$ protein

$21 \mathrm{kDa}$ protein

CDV3 $5 \mathrm{kDa}$ protein

CDV3 isoform 2 of protein CDV3 homolog

FAU 40S ribosomal protein $\mathrm{S} 30$

FAU ubiquitin-like protein fubi and ribosomal protein S30 precursor

ATP5A1 ATP synthase subunit $\alpha$, mitochondrial

AK2 isoform 1 of adenylate kinase isoenzyme 2, mitochondrial

LDHA isoform 2 of L-lactate dehydrogenase A chain

RBM3 putative RNA-binding protein 3

RBM3 putative uncharacterized protein RBM3

MRCL3 myosin regulatory light chain MRCL3 variant

MRLC2 myosin regulatory light chain MRLC2

CSRP1 cysteine and glycine-rich protein 1

SCAMP3 isoform 1 of secretory carrier-associated membrane protein 3

RPS21 40S ribosomal protein S21

PPP1R12A isoform 1 of protein phosphatase 1 regulatory subunit $12 \mathrm{~A}$

LOC653665 similar to mCG4465, partial

LOC641293 similar to 60S ribosomal protein L21

LOC653156 similar to hCG1782414 isoform 2

EIF4A1 eukaryotic initiation factor 4A-I

ALB isoform 1 of serum albumin

HSPD1 $60 \mathrm{kDa}$ heat shock protein, mitochondrial

PPIA peptidyl-prolyl cis-trans isomerase A

STMN1 stathmin

STMN1 stathmin 1 variant

NPM1 isoform 1 of nucleophosmin

cDNA FLJ52243, highly similar to heat-shock protein $\beta$-1

TUBB2C tubulin $\beta$-2C chain

cDNA FLJ11352 fis, clone HEMBA1000020, highly similar to tubulin $\beta-2 \mathrm{C}$

NCL nucleolin

ACTA1 actin $\alpha 1$ skeletal muscle protein

ACTA2 actin, aortic smooth muscle

ACTB cDNA FLJ52842, highly similar to actin, cytoplasmic 1 
Table I. Continued.

\begin{tabular}{|c|c|}
\hline iTRAQ ratio & Protein name \\
\hline 0.666 & ACTB $11 \mathrm{kDa}$ protein \\
\hline 0.782 & PRSS1 trypsin-1 \\
\hline 0.670 & ACTA2 ACTA2 protein (fragment) \\
\hline 0.670 & A26C1B ANKRD26-like family C members 1B \\
\hline 0.670 & LOC653269 similar to protein expressed in prostate, ovary, testis and placenta \\
\hline 0.670 & A26C1A isoform 1 of ANKRD26-like family C member $1 \mathrm{~A}$ \\
\hline 0.568 & TUBB4Q putative tubulin $\beta-4 q$ chain \\
\hline 0.568 & TUBB4Q tubulin, $\beta$ polypeptide 4 , member $Q$ \\
\hline 0.568 & RP11-631M21.2 tubulin $\beta-8$ chain \\
\hline 0.630 & TUBB4 tubulin $\beta-4$ chain \\
\hline 0.774 & Mitochondrial heat shock $60 \mathrm{kDa}$ protein 1 variant 1 \\
\hline 0.813 & AHNAK neuroblast differentiation-associated protein AHNAK \\
\hline 0.935 & TPM4 isoform 1 of tropomyosin $\alpha-4$ chain \\
\hline 0.935 & TPM4 cDNA FLJ52936, weakly similar to tropomyosin $\alpha-4$ chain \\
\hline 0.846 & TPM3 isoform 2 of tropomyosin $\alpha-3$ chain \\
\hline 0.912 & HNRNPA1 isoform A1-B of heterogeneous nuclear ribonucleoprotein A1 \\
\hline 0.912 & LOC645691 similar to heterogeneous nuclear ribonucleoprotein A1 \\
\hline 0.912 & LOC645691 similar to heterogeneous nuclear ribonucleoprotein A1 \\
\hline 0.912 & HNRNPA1 isoform 2 of heterogeneous nuclear ribonucleoprotein A1 \\
\hline 0.912 & cDNA FLJ51587, highly similar to heterogeneous nuclear ribonucleoprotein \\
\hline 0.960 & MIF; LOC284889 macrophage migration inhibitory factor \\
\hline 0.857 & TPM3 tropomyosin 3 \\
\hline 0.964 & RPS10 40S ribosomal protein S10 \\
\hline 0.964 & LOC388885 similar to ribosomal protein S10 \\
\hline 0.871 & PDIA3 protein disulfide-isomerase A3 \\
\hline 0.821 & HNRNPM isoform 2 of heterogeneous nuclear ribonucleoprotein $\mathrm{M}$ \\
\hline 0.985 & TPM2 isoform 2 of tropomyosin $\beta$ chain \\
\hline 0.985 & TPM2 isoform 3 of tropomyosin $\beta$ chain \\
\hline 0.985 & TPM1 tropomyosin $1 \alpha$ variant 6 \\
\hline 0.985 & TPM1 tropomyosin $1 \alpha$ chain isoform 7 \\
\hline 0.985 & TPM3 tropomyosin 3 isoform 1 \\
\hline 0.985 & TPM1 isoform 4 of tropomyosin $\alpha-1$ chain \\
\hline 0.985 & TPM1 tropomyosin isoform \\
\hline 0.983 & KHSRP ısoform 2 of Far upstream element-binding protein 2 \\
\hline 0.983 & cDNA FLJ51330, highly similar to Far upstream element-binding protein 2 \\
\hline 0.900 & RPL23A protein \\
\hline 0.765 & LOC654188 similar to peptidylprolyl isomerase A-like \\
\hline 0.765 & PPIAP19 similar to peptidylprolyl isomerase A-like \\
\hline 0.900 & LOC130773 similar to ribosomal protein L23a \\
\hline 0.744 & EIF4B cDNA FLJ59405, highly similar to eukaryotic translation initiation \\
\hline 0.744 & EIF4B cDNA FLJ54492, highly similar to eukaryotic translation initiation \\
\hline 0.744 & cDNA FLJ59206, highly similar to eukaryotic translation initiation factor \\
\hline 0.903 & YAP1 65 kDa Yes-associated protein \\
\hline 0.903 & YAP1 Yes-associated protein 1, $65 \mathrm{kDa}$ isoform 1 \\
\hline 0.850 & cDNA FLJ56081, highly similar to lamin-A/C \\
\hline 0.850 & LMNA progerin \\
\hline 0.850 & LMNA isoform A of lamin-A/C \\
\hline 0.914 & AHNAK putative uncharacterized protein AHNAK \\
\hline 0.629 & HIST1H1C histone H1.2 \\
\hline 0.629 & HIST1H1D histone H1.3 \\
\hline
\end{tabular}


Table I. Continued.

\section{iTRAQ ratio}

Protein name
0.629

0.852

0.738

0.889

0.975

0.911

0.269

0.269

0.862

0.844

0.850

0.456

0.456

0.783

0.565

0.948

0.432
HIST1H1E histone H1.4

TAGLN2 $21 \mathrm{kDa}$ protein

C1QBP complement component $1 \mathrm{Q}$ subcomponent-binding protein, mitochondrial SF3B2 splicing factor 3B subunit 2

HSPA9 stress-70 protein, mitochondrial

SF3B2 putative uncharacterized protein DKFZp781L0540 (fragment)

HIST2H2BE histone H2B type 2-E

HIST2H3PS2 histone H2B

HSPA5 HSPA5 protein

MKI67 isoform long of antigen KI-67

PRKCSH cDNA FLJ59211, highly similar to glucosidase 2 subunit $\beta$

TPM3 tropomyosin 3

LOC147804 tropomyosin 3 pseudogene

STMN1 stathmin 1/oncoprotein 18

ACTG1 actin, cytoplasmic 2

NCL cDNA FLJ10452 fis, clone NT2RP1000966, highly similar to nucleolin PRDX1 peroxiredoxin-1

Ratios are relative to 114 (uniduced) and reagent 117 (induced) $>1.0$ upregulated and $<1.0$ downregulated upon PDEF induction.
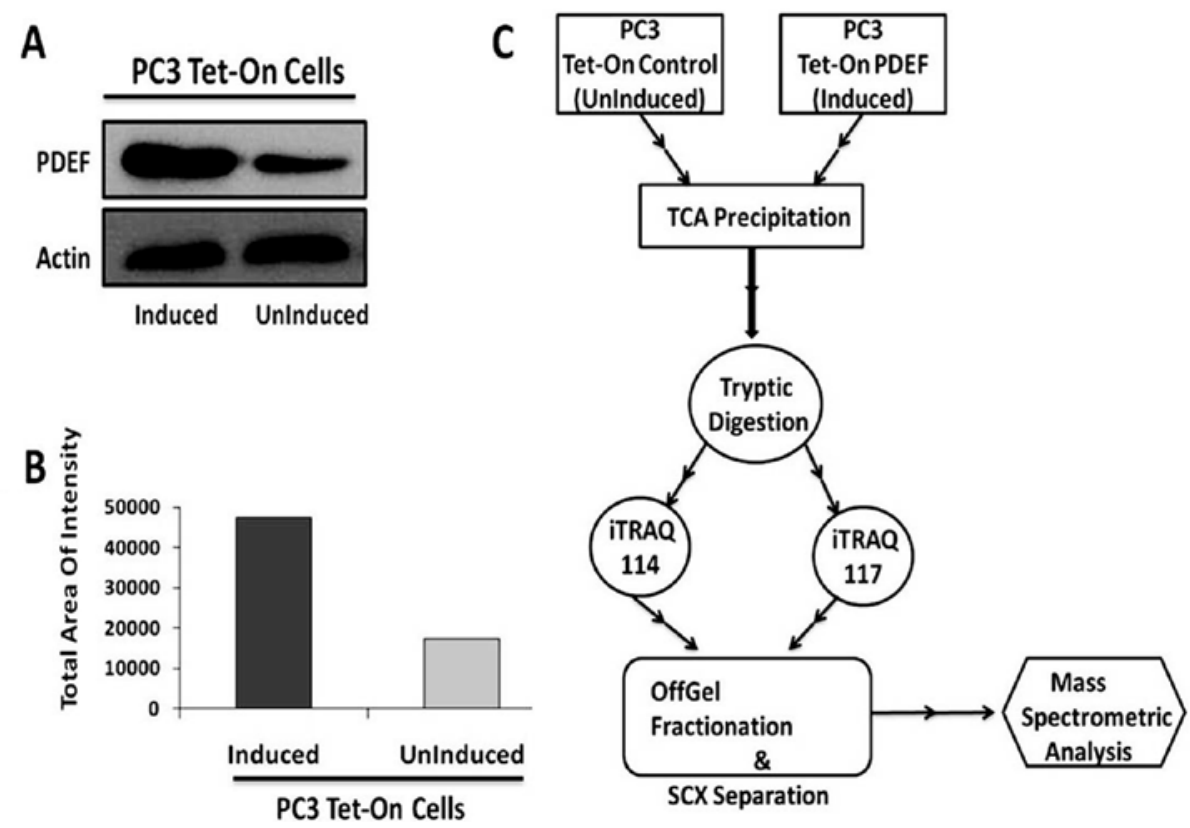

Figure 1. Western blot analysis of PDEF overexpression in PC3 cells using a tetracycline-inducible system (Tet-On). (A) PC3 Tet-On cells were induced with $1 \mu \mathrm{g} / \mathrm{ml}$ doxycycline (DOX) for $24 \mathrm{~h}$. Blot was probed with polyclonal antibody specific to PDEF and actin (as loading control). (B) 2.7-fold increase in area of intensity was observed upon quantitation of induced and uninduced PC3 Tet-On cells. (C) Overview of the iTRAQ labeling process from sample preparation to fractionation and protein identification.

in comparison to uninduced sample control (Fig. 1B). After confirming the protein expression, uninduced and induced cells were iTRAQ labeled separately as represented schematically in Fig. 1C. We identified 115 proteins from our analysis of the iTRAQ labeling experiment (Table I). All 115 proteins fell into the preset selection criteria set by us and only these proteins were used for any further analysis. Of the 115 proteins identified as differentially expressed, 80 proteins were downregulated and 35 proteins were upregulated upon PDEF induction (Table I). As mascot search returns its ratios relative to 114 , therefore all those proteins $>1.0$ were found upregulated and all the proteins $<1.0$ were found downregulated upon PDEF induction. 

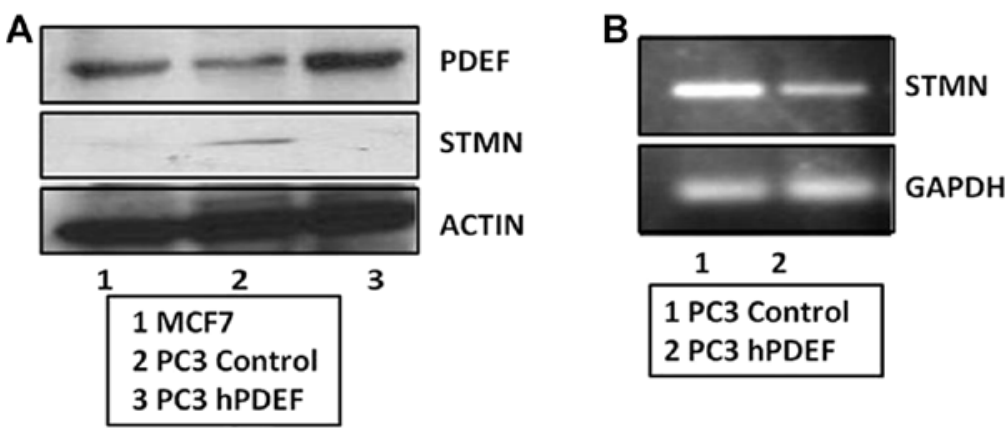

Figure 2. Validation of expression of microtubule (MT)-destabilizing protein STMN upon PDEF induction. (A) Western blot analysis of PC3 pcDNA3.1 hPDEF cells for PDEF and STMN protein expression levels with specific antibodies. MCF7 cell lysate was used as positive control. Actin was used as loading control. (B) RT-PCR analysis of PC3 pcDNA3.1 hPDEF cells for mRNA expression analysis with specific STMN primers. GAPDH was used as the loading control.

Criteria for selection of target protein for further analysis. Our study was based on 3 basic criteria for the selection of target protein for further study in relation to PDEF in the process of prostate cancer progression: i) proteins with the ITRAQ ratio $(>1.0$ or $<1.0)$, ii) number of times the protein showed up in the analyzed sample sets ( $>2$ times) and iii) role in the prostate cancer process (based on the literature).

Based on the above criteria, we first analyzed the differentially expressed proteins with the iTRAQ ratios $>1.0$ and $<1.0$. 80 proteins were downregulated with a ratio below 1.0 and 35 were upregulated with a ratio above 1.0 (Table I). Most of the proteins were found downregulated $(<1.0)$ upon PDEF induction in PC3 prostate cancer cells.

Based on our second selection criteria, 11 out of the 115 proteins showed differential expression three times or more ( $>3$ times) in the analyzed sample sets (Table I). The proteins were heterogeneous nuclear ribonucleoprotein (HNRNPU), tubulin, heat shock proteins (HSP), peptidyl propyl isomerase, stathmin, actin, tropomyosin (TPM), cortactin (CTTN), ribosomal protein, eukaryotic translation initiation factor (EIF) and histones. In order to further narrow down our search, we decided to exclude those proteins which were associated to any isoforms or were highly similar to the identified proteins. Also proteins that were identified as chains ( $\alpha$ or $\beta$ ), types (type $2 \mathrm{E}$ and B), subunits such as S21, S10 or proteins that came as forms of the identified protein (such as mitochondrial and cytoplasmic) were all excluded from our study. Based on our exclusion criteria we found only one protein STMN. STMN was found differentially expressed multiple times and was taken into consideration for further analysis.

STMN was found extensively studied in relation to the cancer progression process $(27,28)$ and in many studies directly linked to prostate cancer $(29,30)$. STMN in our study was found downregulated multiple times $(0.829,0.829$ and 0.783$)$ as seen in Table I. Keeping all these considerations in mind, we focused our research primarily on the association of STMN and PDEF in the process of prostate cancer progression.

Stathmin, a differentially expressed protein. Stathmin (STMN) has been extensively studied in cancer progression, specifically in relation to prostate cancer $(29,30)$. STMN, also known as p17, p18, p19, 19k, metablastin, oncoprotein 18, LAP18, Op18, is a $19-\mathrm{kDa}$ cytosolic protein. It was the first discovered member of family of MT-destabilizing phosphoproteins

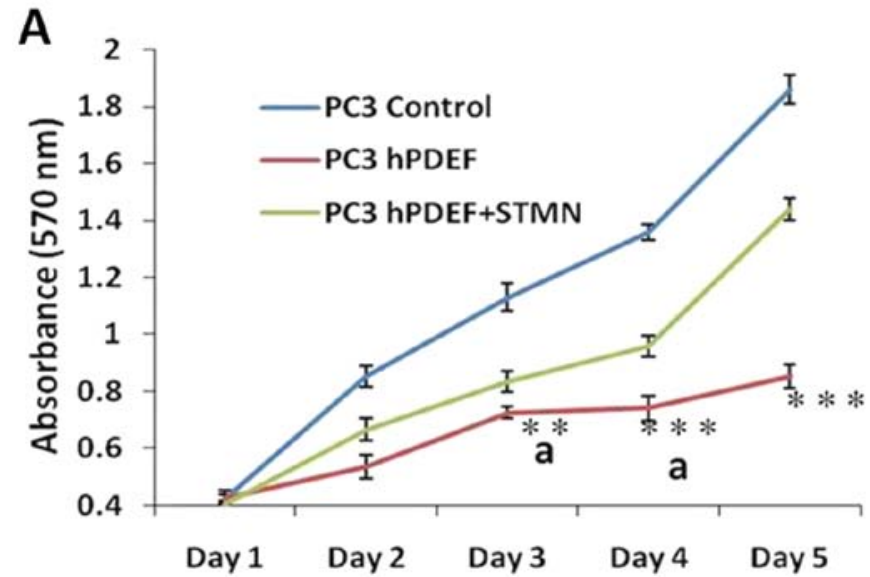

B

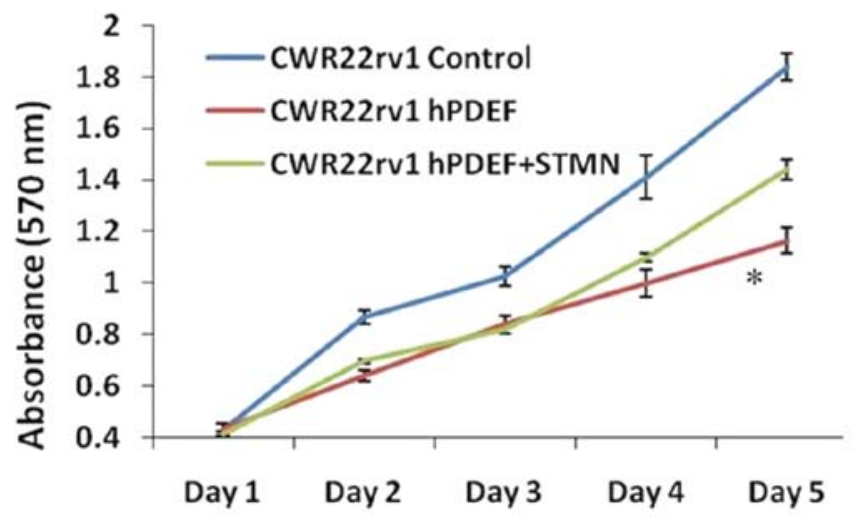

Figure 3. Expression of STMN reverses in vitro cell growth of PC3 and CWR22rv1 hPDEF cells. In vitro cell growth of various PC3 cell lines (A) and CWR22rv1 cell lines (B) a, PC3/CWR22rv1 hPDEF vs. PC3/CWR22rv1 $\mathrm{hPDEF}+\mathrm{STMN}$ on respective days. ${ }^{* *} \mathrm{p}<0.01 ;{ }^{* * *} \mathrm{p}<0.001$.

$(31,32)$. This protein was critically involved in the assembly and disassembly of the mitotic spindle involved in the progression through the cell cycle (29,33). Western blot analysis (Fig. 2A) and mRNA expression level (Fig. 2B) of PC3 control and hPDEF cell lysates confirmed the downregulation of STMN in PC3 hPDEF cells.

Relevance of STMN to the prostate cancer progression process. To further evaluate the relevance of STMN and PDEF associ- 
A
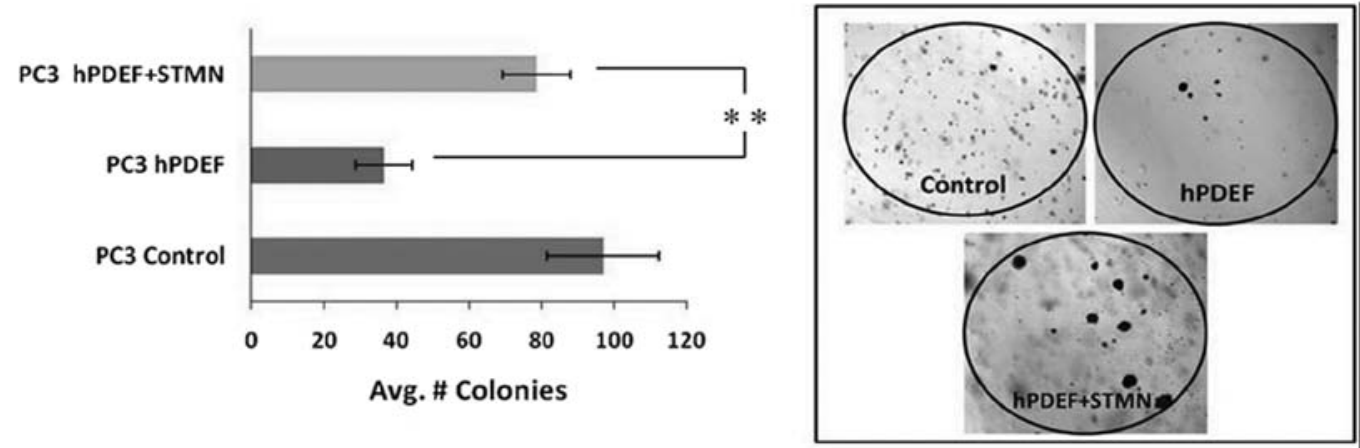

B
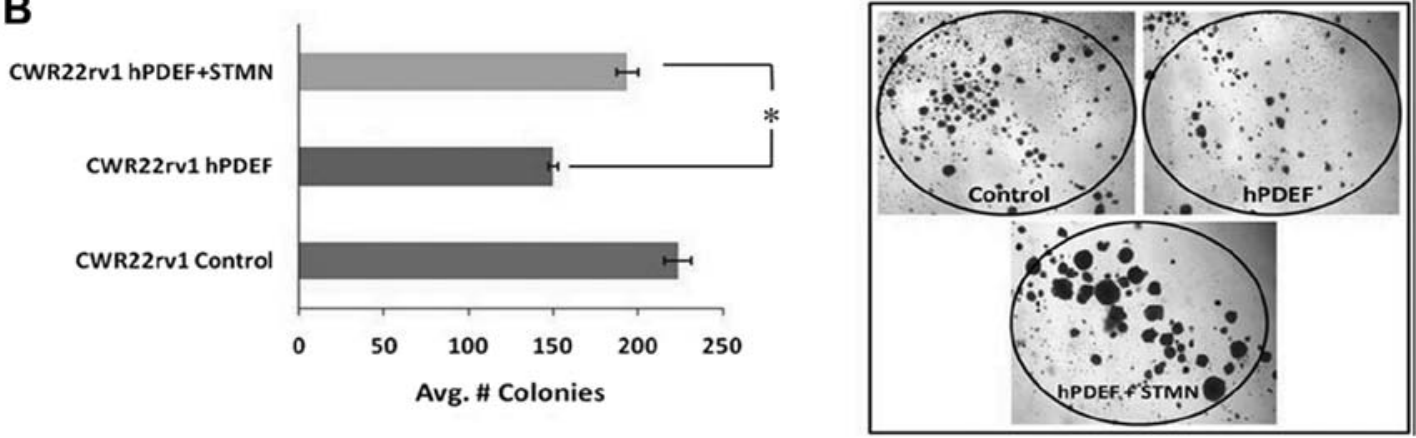

Figure 4. STMN re-expression increases in vitro three-dimensional soft agar colony formation in PC3 and CWR22rv1 cell lines. In vitro anchorage independent growth assay of various PC3 cell lines (A) and various CWR22rv1 cell lines (B). It is worth noting that the hPDEF+STMN colonies are larger in comparison to others in both PC3 and CWR22rv1 cells. PC3/CWR22rv1 hPDEF vs. PC3/CWR22rv1 hPDEF+STMN *"p $<0.01$, "p $<0.05$.

ation during the process of prostate cancer progression. The proliferative, migratory and colony forming potential of prostate cancer cells were evaluated in PC 3 cells as well as CWR22rv1, another prostate cell line. Both PC3 and CWR22rv1 stable transfectants showed a significant change in cells overexpressing hPDEF+STMN in comparison to hPDEF cells alone. PC 3 and CWR22rv1 hPDEF cells display a tumor suppressing effect, which is reversed in the presence of STMN. Our previous in vitro and in vivo data prove that PDEF is a tumor suppressor in breast cancer cells. We showed that PDEF-expressing cells block the cell growth rate, and this retardation was reversed when PDEF expression was silenced with PDEF-specific small interfering RNA (16). Upon STMN expression PDEF overexpressing cells show increased growth potential in an MTT proliferation assay in both PC3 (Fig. 3A) and CWR22rv1 (Fig. 3B) prostate cells. The increase in growth rate was $\sim 2$ times more than that of PC3 hPDEF cells, but was lower than the PC3 prostate cancer cells. CWR22rv1 prostate cancer cells also showed similar reversal effect in CWR22rv1 hPDEF+STMN cells but was not as significant as PC3 prostate cancer cells. This difference in both the prostate cancer cell lines could be attributed to the aggressiveness of the cell lines. Soft agar colony formation potential of hPDEF cells in both prostate cancer cell types was significantly increased (PC3, $\mathrm{p}<0.015$; CWR22rv1, $\mathrm{p}<0.036$ ) in the presence of STMN in hPDEF cells (Fig. 4). The colonies formed were distinctly larger in the presence of STMN in comparison to all other cell types. This result clearly demonstrates the anchorageindependent growth potential. In the presence of STMN, the colonies formed faster and were significantly larger in both the PC3 and CWR22rv1 hPDEF+STMN cells than in the PC3/
CWR22rv1 hPDEF cells alone. Clearly showing the importance of the association of STMN and PDEF in the cancer progression process, as anchorage-independence is one of the key characteristics of cancer cells. Anchorage-independence helps cancer cells to grow and distribute themselves throughout the body. The migratory potential was significantly increased in the presence of STMN in PC3 $(\mathrm{p}<0.0055)$ and CWR222rv1 $(p<0.023)$ cells (Fig.5). The migratory potential of PC3 hPDEF+STMN cells increased by $\sim 50 \%$ in comparison to PC3 hPDEF cells. In CWR22rv1 hPDEF+STMN cells the migratory potential was increased by $\sim 30-35 \%$. STMN in the presence of hPDEF showed a significant change in the key steps of the cancer progression process such as proliferation, colony formation and migration. The above results from the in vitro functional assays clearly demonstrate the change due to the introduction of STMN in hPDEF in PC3/CWR22rv1 cells. It also shows the importance of the association of STMN and PDEF in the cancer progression process. The results from the above described in vitro functional assays have been summarized graphically in Fig. 6. The data clearly point to the importance of PDEF and STMN association in the process of prostate cancer progression.

\section{Discussion}

The Ets family of proteins consists of a large number of evolutionarily conserved transcription factors. Many of Ets factors have been implicated in key steps of the tumor progression process $(6,11)$. Extensive studies have been done with Ets family proteins focusing on their biochemical properties and cellular functions $(4,34)$. Following the discovery of PDEF in 
A

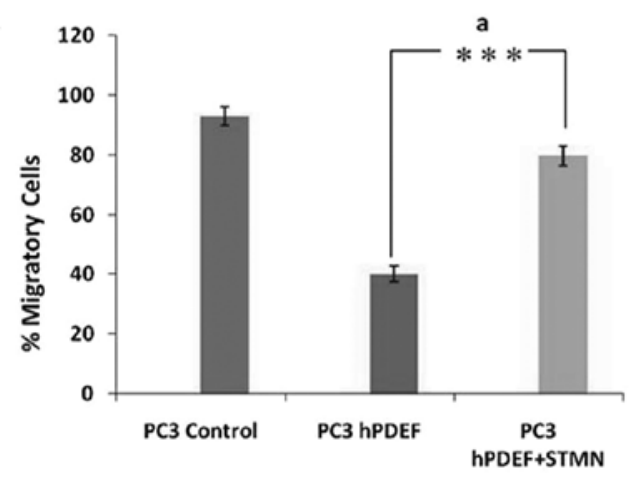

B

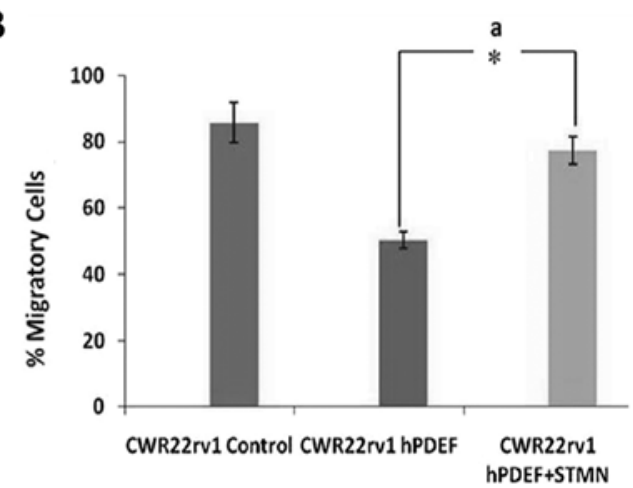

Figure 5. STMN expression increased the migratory potential of PC3 and CWR22rv1 hPDEF cell lines. In vitro wound healing assay, analyzing the migratory potential of various PC3 cell lines (A) and CWR22rv1 cell lines (B). STMN re-expression significantly increases in vitro migration. a, PC3/CWR22rv1 hPDEF vs. PC3/CWR22rv1 hPDEF+STMN ${ }^{* * *}$ p $<0.001,{ }^{*} \mathrm{p}<0.05$.

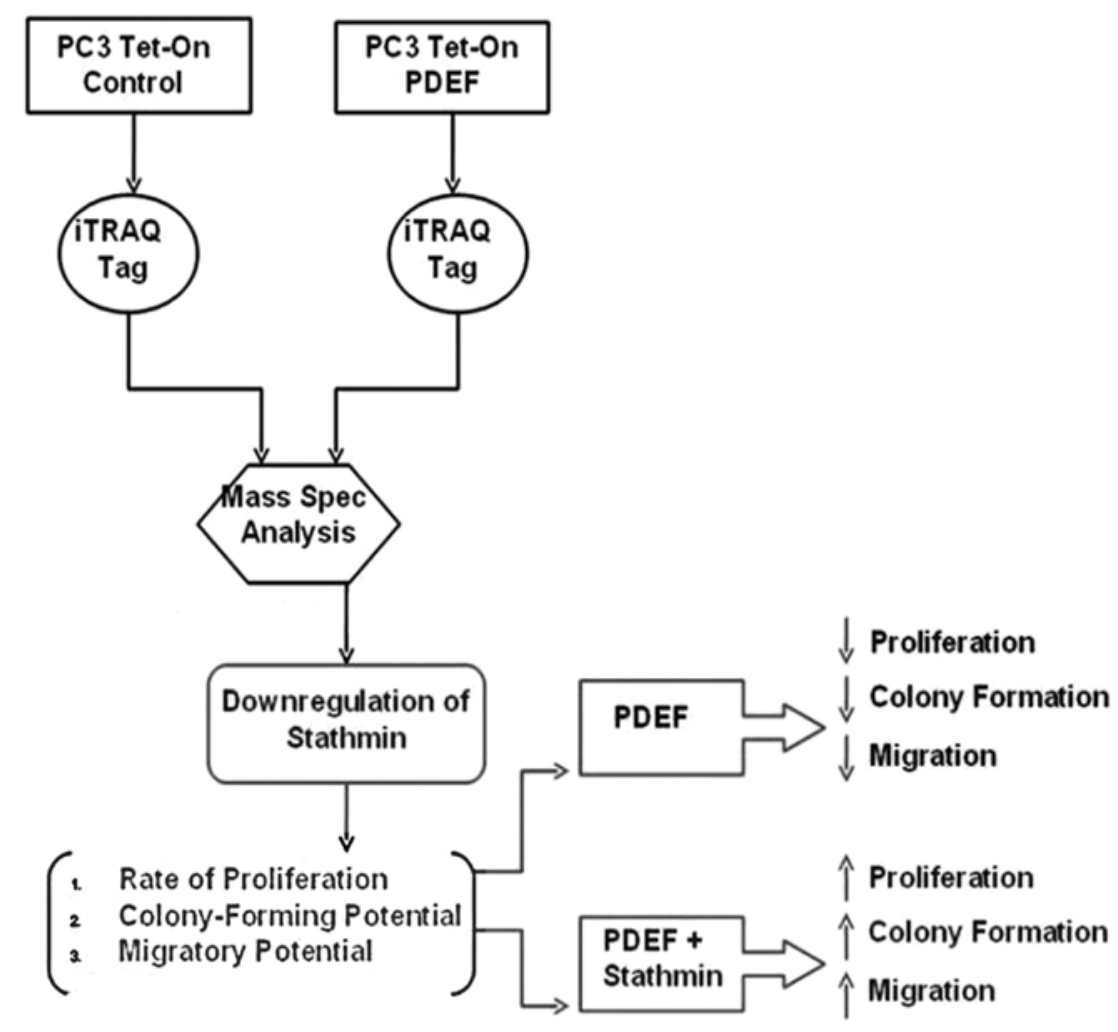

Figure 6. Graphical abstract: overview of the role of stathmin in PDEF overexpressing prostate cells.

year 2000 (35), much work has been done to understand the role of PDEF in the process of normal growth and development and in cancer progression. PDEF, a key Ets transcription factor has been studied by various groups involved in the process of tumor progression $(17,36,37)$.

In the present study, we used iTRAQ labeling and proteomic approaches to analyze the differentially expressed proteins in prostate cancer cells upon PDEF induction and confirmed their association using in vitro functional assays. We have not only used a different approach to identify PDEF regulated proteins but also analyzed the effect of PDEF and STMN association on prostate tumor progression. We are the first to use iTRAQ technology to explore the PDEF proteome in cancer cells and verify our results with in vitro assays. We used iTRAQ technology as it has significant advantages over other methods coupled with mass spectrometric analysis. The major advantage of iTRAQ labeling is the capability of multiplexing different samples at the same time to avoid experimental bias. Another positive aspect includes unbiased peptide labeling, as the peptide reactive group is able to bind with all free amino groups to the peptide in the sample $(20,38,39)$.

We identified various proteins as differentially expressed from the analyzed experimental sets. Many of the proteins identified were downregulated upon PDEF induction. STMN was selected as the candidate protein for further analysis based on three set selection criteria. Firstly, STMN was identified 
with an iTRAQ ratio $(<1.0)$, secondly STMN showed up multiple times ( 3 times) in all analyzed sample sets and lastly extensive research was done with STMN in prostate cancer, including designing therapeutic targets. Finally one other reason in consideration was the fact that STMN gene reveals multiple transcription factor recognition sequences: two AP-2 sites, five GC boxes and four E2F sites $(40,41)$. The presence of multiple transcription factor recognition sequences gives us more confidence of the possibility of a site for PDEF binding on STMN.

STMN is overexpressed in many human malignancies including leukemia (42), lymphoma (43), neuroblastoma (44); ovarian (45), prostatic $(46)$, breast $(47,48)$ and lung cancer $(49)$. In many of these cancers, high STMN expression correlates with bad prognosis. It is upregulated in normal proliferating cell lines and also rarely upregulated in non-proliferating cell lines with the exception of neurons (50). STMN expression is highest when the prostate is undergoing morphogenesis or tumorigenesis and these processes may be regulated through differential phosphorylation (30). One of key function of STMN is to alter microtubule dynamics based on the need of the cells. STMN plays a role in both the overexpression and inhibition of expression, which could result in mitotic arrest due to microtubule altering effects providing an attractive therapeutic target in cancer therapy $(29,51,52)$.

STMN has been extensively studied in relation to prostate cancer, exerting a profound influence on cell proliferation, differentiation and cell motility. Mistry et al has shown a dramatic dose-dependent growth inhibition in $\mathrm{LNCaP}$ prostate cancer cells transduced with anti-STMN adenovirus (53). STMN has provided an attractive molecule to target for cancer therapies that can disrupt the mitotic apparatus (29). Research showed that the anti-STMN ribozyme and low non-inhibitory concentrations of Taxol and etoposide had a profound synergistic inhibitory effect on proliferation, clonogenicity and induction of apoptosis. This was found to be very relevant for the treatment of prostate cancer as both Taxol and etoposide are agents that have been used earlier to treat this disease (53).

Our in vitro functional data showed that STMN was not seen in PC3 hPDEF cells in comparison to control cells with an antibody specific to STMN (Fig. 2A). PC3 hPDEF cells had a protein expression level 3-fold greater than the PC3 control cells (Fig. 1A and B). RT-PCR analysis showed that the mRNA expression level of STMN using specific primers was decreased $~ 1.5$-fold in PC3 hPDEF cells in comparison to controls (Fig. 2B). This result was in consensus with our iTRAQ data that showed decreased expression of STMN upon PDEF induction.

We were able to further analyze the effect of STMN in the presence of PDEF induction by in vitro functional assays. In vitro MTT proliferation assay, anchorage-independent soft agar colony formation and wound healing migration assay clearly showed that PC3 and CWR22rv1 hPDEF cells were able to inhibit proliferation (Fig. 3), colony formation (Fig. 4) and migration of prostate cancer cells (Fig. 5). Upon STMN expression in the hPDEF cells, all important steps of the cancer progression process were almost completely reversed back to that of the control (Fig. 6). STMN expression significantly increased proliferation, colony forming potential and migratory effects of PC3/CWR22rv1 hPDEF cells. Interestingly our data clearly suggested an association of STMN and PDEF with prostate cancer cells. Though we did see an almost complete reversal of prostate cells upon STMN expression, it was not $100 \%$ and this difference could be attributed to other PDEFassociated proteins that may play role in the process either along with STMN or alone.

STMN has proven to be an interesting protein that was identified multiple times as downregulated upon PDEF induction. The data obtained from functional assays further confirm the findings and provide new insights into the role of PDEF in regulating the prostate cancer process associated with STMN. PDEF has been studied in our laboratory as a tumor suppressor (16) and with the use of iTRAQ labeling and mass spectrometric analysis techniques we were able to shed light on a possible role of PDEF in prostate cancer cells.

Because of the interesting in vitro functional data that support our iTRAQ-MS research it would be worth studying the in-depth association of STMN with PDEF at a molecular level to better understand the disease process. Validating other identified significantly expressed proteins in the presence or absence of STMN upon PDEF induction could explain why there was not a complete reversal upon STMN expression seen in our in vitro studies.

Compelling evidence from the literature has shown that STMN is a target for anti-cancer therapeutics. Our findings suggest that PDEF may be used to suppress STMN oncogene expression and be used for the development of effective therapeutic strategies for the treatment of prostate cancer. The therapeutic strategies designed can also be used in other cancers.

\section{Acknowledgements}

The authors would like to thank Dr George P. Tuszynski for his helpful discussion. This study was supported by an NIH P50 CA090386 pilot project fund and a PCF fund to M.Z.

\section{References}

1. www.cancer.org, 2010.

2. Nash AF and Melezinek I: The role of prostate specific antigen measurement in the detection and management of prostate cancer. Endocr Relat Cancer 7: 37-51, 2000.

3. Klotz L: Active surveillance for prostate cancer: for whom? J Clin Oncol 23: 8165-8169, 2005.

4. Sementchenko VI and Watson DK: Ets target genes: past, present and future. Oncogene 19: 6533-6548, 2000

5. Oikawa T: ETS transcription factors: possible targets for cancer therapy. Cancer Sci 95: 626-633, 2004.

6. Seth A and Watson DK: ETS transcription factors and their emerging roles in human cancer. Eur J Cancer 41: 2462-2478, 2005.

7. Galang CK, Muller WJ, Foos G, Oshima RG and Hauser CA: Changes in the expression of many Ets family transcription factors and of potential target genes in normal mammary tissue and tumors. J Biol Chem 279: 11281-11292, 2004.

8. Hsu T, Trojanowska M and Watson DK: Ets proteins in biological control and cancer. J Cell Biochem 91: 896-903, 2004.

9. Feldman RJ, Sementchenko VI and Watson DK: The epithelialspecific Ets factors occupy a unique position in defining epithelial proliferation, differentiation and carcinogenesis. Anticancer Res 23: 2125-2131, 2003.

10. Li R, Pei H and Watson DK: Regulation of Ets function by protein-protein interactions. Oncogene 19: 6514-6523, 2000.

11. Papas TS, Blair DG, Watson DK, Yuan CC, Ruscetti SK, Fujiwara S, Seth AK, Fisher RJ, Bhat NK, Mavrothalassitis G, et al: The ETS family of genes: structural analysis, gene products, and involvement in neoplasia and other pathologies. Prog Clin Biol Res 360: 137-168, 1990. 
12. Oettgen P, Finger E, Sun Z, Akbarali Y, Thamrongsak U, Boltax J, Grall F, Dube A, Weiss A, Brown L, Quinn G, Kas K, Endress G, Kunsch C and Libermann TA: PDEF, a novel prostate epithelium-specific ets transcription factor, interacts with the androgen receptor and activates prostate-specific antigen gene expression. J Biol Chem 275: 1216-1225, 2000.

13. Ghadersohi A and Sood AK: Prostate epithelium-derived Ets transcription factor mRNA is overexpressed in human breast tumors and is a candidate breast tumor marker and a breast tumor antigen. Clin Cancer Res 7: 2731-2738, 2001

14. Sood AK, Saxena R, Groth J, Desouki MM, Cheewakriangkrai C, Rodabaugh KJ, Kasyapa CS and Geradts J: Expression characteristics of prostate-derived Ets factor support a role in breast and prostate cancer progression. Hum Pathol 38: 1628-1638, 2007.

15. Findlay VJ, Turner DP, Moussa O and Watson DK: MicroRNAmediated inhibition of prostate-derived Ets factor messenger RNA translation affects prostate-derived Ets factor regulatory networks in human breast cancer. Cancer Res 68: 8499-8506, 2008.

16. Schaefer JS, Sabherwal Y, Shi HY, Sriraman V, Richards J, Minella A, Turner DP, Watson DK and Zhang M: Transcriptional regulation of p21/CIP1 cell cycle inhibitor by PDEF controls cell proliferation and mammary tumor progression. J Biol Chem 285: 11258-11269, 2010.

17. Ghadersohi A, Odunsi K, Lele S, Collins Y, Greco WR, Winston J, Liang P and Sood AK: Prostate derived Ets transcription factor shows better tumor-association than other cancer-associated molecules. Oncol Rep 11: 453-458, 2004.

18. Gu X, Zerbini LF, Out HH, Bhasin M, Yang Q, Joseph MG Grall F, Onatunde T, Correa RG and Libermann TA: Reduced PDEF expression increases invasion and expression of mesenchymal genes in prostate cancer cells. Cancer Res 67: 4219-4226, 2007.

19. Turner DP, Moussa O, Sauane M, Fisher PB and Watson DK: Prostate-derived ETS factor is a mediator of metastatic potential through the inhibition of migration and invasion in breast cancer. Cancer Res 67: 1618-1625, 2007.

20. Zieske LR: A perspective on the use of iTRAQ reagent technology for protein complex and profiling studies. J Exp Bot 57: 1501-1508, 2006.

21. Zahedi RP, Sickmann A, Boehm AM, Winkler C, Zufall N, Schonfisch B, Guiard B, Pfanner N and Meisinger C: Proteomic analysis of the yeast mitochondrial outer membrane reveals accumulation of a subclass of preproteins. Mol Biol Cell 17: $1436-1450,2006$

22. Choe L, D'Ascenzo M, Relkin NR, Pappin D, Ross P, Williamson B, Guertin S, Pribil P and Lee KH: 8-Plex quantitation of changes in cerebrospinal fluid protein expression in subjects undergoing intravenous immunoglobulin treatment for Alzheimer's disease. Proteomics 7: 3651-3660, 2007.

23. Ross PL, Huang YN, Marchese JN, Williamson B, Parker K, Hattan S, Khainovski N, Pillai S, Dey S, Daniels S, Purkayastha S Juhasz P, Martin S, Bartlet-Jones M, He F, Jacobson A and Pappin DJ: Multiplexed protein quantitation in saccharomyces cerevisiae using amine-reactive isobaric tagging reagents. Mol Cell Proteomics 3: 1154-1169, 2004

24. Cho JY, Lee M, Ahn JM, Park ES, Cho JH, Lee SJ, Kim BG, Heo SH, Park HJ, Zerbini LF, Hwang D and Libermann TA: Proteomic analysis of a PDEF Ets transcription factor-interacting protein complex. J Proteome Res 8: 1327-1337, 2009.

25. Gossen $\mathrm{M}$ and Bujard $\mathrm{H}$ : Tight control of gene expression in mammalian cells by tetracycline-responsive promoters. Proc Natl Acad Sci USA 89: 5547-5551, 1992.

26. Warren CM, Geenen DL, Helseth JDL, $\mathrm{Xu} \mathrm{H}$ and Solaro RJ: Sub-proteomic fractionation, iTRAQ, and OFFGEL-LC-MS/MS approaches to cardiac proteomics. J Proteomics 73: 1551-1561, 2011.

27. Jeon TY, Han ME, Lee YW, Lee YS, Kim GH, Song GA Hur GY, Kim JY, Kim HJ, Yoon S, Baek SY, Kim BS, Kim JB and Oh SO: Overexpression of stathmin 1 in the diffuse type of gastric cancer and its roles in proliferation and migration of gastric cancer cells. Br J Cancer 102: 710-718, 2006.

28. Alli E, Yang JM and Hait WN: Silencing of stathmin induces tumor-suppressor function in breast cancer cell lines harboring mutant p53. Oncogene 26: 1003-1012, 2006.

29. Mistry SJ and Atweh GF: Therapeutic interactions between stathmin inhibition and chemotherapeutic agents in prostate cancer. Mol Cancer Ther 5: 3248-3257, 2006.

30. Ghosh R, Gu G, Tillman E, Yuan J, Wang Y, Fazli L, Rennie PS and Kasper S: Increased expression and differential phosphorylation of stathmin may promote prostate cancer progression. Prostate 67: $1038-1052,2007$
31. Larsson N, Marklund U, Gradin HM, Brattsand G and Gullberg M: Control of microtubule dynamics by oncoprotein 18: dissection of the regulatory role of multisite phosphorylation during mitosis. Mol Cell Biol 17: 5530-5539, 1997.

32. Beretta L, Dobránsky T and Sobel A: Multiple phosphorylation of stathmin. Identification of four sites phosphorylated in intact cells and in vitro by cyclic AMP-dependent protein kinase and p34cdc2. J Biol Chem 268: 20076-20084, 1993.

33. Desai A and Mitchison TJ: Microtubule polymerization dynamics. Annu Rev Cell Dev Biol 13: 83-117, 1997.

34. Watson DK, Ascione R and Papas TS: Molecular analysis of the ets genes and their products. Crit Rev Oncog 1: 409-436, 1990.

35. Yamada N, Tamai Y, Miyamoto H and Nozaki M: Cloning and expression of the mouse Pse gene encoding a novel Ets family member. Gene 241: 267-274, 2000.

36. Gunawardane RN, Sgroi DC, Wrobel CN, Koh E, Daley GQ and Brugge JS: Novel role for PDEF in epithelial cell migration and invasion. Cancer Res 65: 11572-11580, 2005.

37. Feldman RJ, Sementchenko VI, Gayed M, Fraig MM and Watson DK: Pdef expression in human breast cancer is correlated with invasive potential and altered gene expression. Cancer Res 63: 4626-4631, 2003.

38. Lin WT, Wu KP, Hung WN, Yian YH, Chen YR, Chen YJ, Sung TY and Hsu WL: MassTRAQ: a fully automated tool for iTRAQ-labeled protein quantification. 2005 IEEE Computational System Bioinformatics Conference Workshops and Poster Abstracts pp157-158, 2005.

39. Lacerda CM, Xin L, Rogers I and Reardon KF: Analysis of iTRAQ data using Mascot and Peaks quantification algorithms. Brief Funct Genomic Proteomic 7: 119-126, 2008.

40. Cassimeris L: The oncoprotein 18/stathmin family of microtubule destabilizers. Curr Opin Cell Biol 14: 18-24, 2002.

41. Gavet O, Ozon S, Manceau V, Lawler S, Curmi P and Sobel A: The stathmin phosphoprotein family: intracellular localization and effects on the microtubule network. J Cell Sci 111: 3333-3346, 1998.

42. Hanash SM, Strahler JR, Kuick R, Chu EH and Nichols D: Identification of a polypeptide associated with the malignant phenotype in acute leukemia. J Biol Chem 263: 12813-12815, 1988.

43. Roos G, Brattsand G, Landberg G, Marklund U and Gullberg M: Expression of oncoprotein 18 in human leukemias and lymphomas. Leukemia 7: 1538-1546, 1993.

44. Hailat N Strahler J, Melhem R, et al: N-myc gene amplification in neuroblastoma is associated with altered phosphorylation of a proliferation related polypeptide (Op 18). Oncogene 5: 1615-1618, 1990.

45. Price DK, Ball JR, Bahrani-Mostafavi Z, et al: The phosphoprotein Op 18/stathmin is differentially expressed in ovarian cancer. Cancer Invest 18: 722-730, 2000.

46. Friedrich B, Grönberg H, Landstrom M, Gullberg M and Bergh A: Differentiation-stage specific expression of oncoprotein 18 in human and rat prostatic adenocarcinoma. Prostate 27: 102-109, 1995.

47. Brattsand G: Correlation of oncoprotein 18/stathmin expression in human breast cancer with established prognostic factors. Br J Cancer 83: 311-318, 2000.

48. Bieche I, Lachkar S, Becette V, et al: Overexpression of the stathmin gene in a subset of human breast cancer. Br J Cancer 78: 701-709, 1998.

49. Nishio K, Nakamura T, Koh Y, Kanzawa F, Tamura T and Saijo N: Oncoprotein 18 overexpression increases the sensitivity to vindesine in the human lung carcinoma cells. Cancer 91: 1494-1499, 2001.

50. Ozon S, El Mestikawy S and Sobel A: Differential, regional, and cellular expression of the stathmin family transcripts in the adult rat brain. J Neurosci Res 56: 553-564, 1999.

51. Mistry SJ, Li HC and Atweh GF: Role for protein phosphatases in the cell-cycle-regulated phosphorylation of stathmin. Biochem J 334: 23-29, 1998.

52. Mistry SJ and Atweh GF: Stathmin inhibition enhances okadaic acid-induced mitotic arrest. J Biol Chem 276: 31209-31215, 2001.

53. Mistry SJ, Benham CJ and Atweh GF: Development of ribozymes that target stathmin, a major regulator of the mitotic spindle. Antisense Nucleic Acid Drug Dev 11: 41-49, 2001. 